\title{
De la chirurgie bariatrique à la chirurgie métabolique : vers un nouveau paradigme dans le traitement du diabète de type 2
}

\author{
From bariatric surgery to metabolic surgery: \\ Towards a new paradigm for the management of type 2 diabetes
}

\author{
A.-J. Scheen ${ }^{1}$, J. De Flines ${ }^{1}$, \\ A. De Roover ${ }^{2}$, N. Paquot ${ }^{1}$ \\ ${ }^{1}$ Université de Liège, \\ service de diabétologie, \\ nutrition et maladies métaboliques, \\ Département de médecine, \\ $\mathrm{CHU}$ de Liège, Belgique. \\ 2 Service de chirurgie digestive, \\ Département de chirurgie, \\ $\mathrm{CHU}$ de Liège, Belgique.
}

\begin{abstract}
Résumé
La chirurgie bariatrique induit de nombreuses modifications hormonales qui contribuent à réduire la sensation de faim et à améliorer l'homéostasie glycémique chez le patient diabétique de type 2. La meilleure connaissance de ces mécanismes physiopathologiques, particulièrement bien étudiés en ce qui concerne la dérivation gastrique avec anse de Roux en Y, a conduit à une certaine innovation dans les techniques chirurgicales proposées et à envisager d'utiliser celles-ci chez des patients diabétiques de type 2 sans obésité sévère (indice de masse corporelle $<35 \mathrm{~kg} / \mathrm{m}^{2}$, voire $<30 \mathrm{~kg} / \mathrm{m}^{2}$ ). Ainsi, on pourrait évoluer d'une chirurgie bariatrique classique vers une chirurgie dite métabolique et s'orienter vers un nouveau paradigme dans le traitement du diabète de type 2 . Cette approche innovante, prometteuse mais encore insuffisamment validée, mérite d'être mieux évaluée, dans une approche multidisciplinaire impliquant une collaboration étroite entre chirurgiens digestifs, gastro-entérologues et endocrino-diabétologues.
\end{abstract}

Mots-clés : Chirurgie bariatrique - chirurgie métabolique

- dérivation gastro-intestinale - diabète de type 2 - hormones digestives - obésité.

\section{Summary}

Bariatric surgery induces numerous hormonal changes that could contribute to reduce hunger sensation and improve glucose homeostasis in patients with type 2 diabetes. The better knowledge of these pathophysiological mechanisms, especially well studied with Roux-en Y gastric bypass, resulted in recent innovation in the technical procedures and to propose them to patients with type 2 diabetes but without severe obesity (body mass index $<35 \mathrm{~kg} / \mathrm{m}^{2}$ or even $<30 \mathrm{~kg} / \mathrm{m}^{2}$ ). Therefore, we may progress in a near future from bariatric surgery to a so-called metabolic surgery, which may open a new paradigm for the management of type 2 diabetes. This innovative approach, promising but still insufficiently validated yet, deserves further careful evaluation in a multidisciplinary approach involving digestive surgeons, gastroenterologists, endocrinologists and diabetologists.

Key-words: Bariatric surgery - metabolic surgery - gastric bypass - type 2 diabetes - intestinal hormones - obesity.

\section{André Scheen}

Département de médecine

CHU Sart Tilman (B35)

4000 Liège - Belgique

andre.scheen@chu.ulg.ac.be

\footnotetext{
๑) 2011 - Elsevier Masson SAS - Tous droits réservés.
} 


\section{Introduction}

La chirurgie bariatrique a conquis une place de plus en plus importante dans le traitement du diabète de type 2 (DT2) associé à une obésité sévère ou extrême [1-5]. La place de la chirurgie dans le traitement du DT2 est sans doute appelée à croître dans les prochaines années pour deux raisons essentielles: - la première est l'échec trop souvent rencontré du traitement médical du DT2, surtout associé à un excès pondéral ou une obésité. L'alternative de disposer d'une approche pharmacologique efficace et sûre pour traiter l'excès pondéral ou l'obésité s'est éloignée au cours des dernières années, avec le retrait successif de divers médicaments commercialisés dans cette indication (fenfluramine, rimonabant, sibutramine, benfluorex), laissant un vide quant à une éventuelle approche médicale ;

- la seconde est que les approches chirurgicales se sont diversifiées au cours des dernières années, avec une approche moins traumatisante grâce à la laparoscopie, un meilleur rapport efficacité/sécurité et, surtout, une meilleure compréhension des mécanismes physiopathologiques sous-jacents conduisant à la perte de poids et aux améliorations métaboliques, parfois spectaculaires (rémission du DT2), observées [5, 6]. Le DT2 est fortement associé à l'excès de poids, en particulier à l'obésité abdominale, et à un faible niveau d'aptitude physique $[7,8]$. Plus de $80 \%$ des patients DT2 présentent au moins un excès de poids (indice de masse corporelle, ou IMC, $>25 \mathrm{~kg} / \mathrm{m}^{2}$ ) et nombre d'entre d'eux sont obèses (IMC > $\left.30 \mathrm{~kg} / \mathrm{m}^{2}\right)$. Le traitement pharmacologique du DT2, en particulier lorsqu'il existe une obésité, s'expose à un taux important d'échecs (hémoglobine glyquée, ou $\mathrm{HbA}_{1 \mathrm{c}}$, restant $>7 \%$ dans au moins la moitié des cas). Par ailleurs, la maladie progresse avec le temps en raison d'un épuisement progressif de la réserve insulinosécrétoire. Cette histoire naturelle, d'une part, impose une approche pharmacologique de plus en plus complexe, onéreuse et à risque de manifestations indésirables, et, d'autre part, rend le taux de succès de plus en plus aléatoire, y compris avec l'insulinothérapie, par ailleurs non exempte de complications. Enfin, la plupart des patients avec un DT2 combinent d'autres facteurs de risque cardiovasculaire, dont les plus communément observés sont une hypertension artérielle et une dyslipidémie. Dès lors, ils payent un lourd tribut aux maladies cardiovasculaires (au moins la moitié d'entre eux vont décéder d'une maladie coronaire et environ un quart d'un accident vasculaire cérébral), ce qui impose une nouvelle donne pour la prise en charge de ces patients [9].

La chirurgie bariatrique, en induisant un amaigrissement important, est capable d'améliorer non seulement le contrôle glycémique, mais également les autres facteurs de risque cardiovasculaire [10] ; elle offre donc le potentiel d'améliorer le pronostic cardiovasculaire des patients avec DT2 [1-6]. Actuellement, les recommandations internationales reconnaissent que la présence d'un DT2 doit amener à considérer le recours à la chirurgie bariatrique lorsque I'IMC est $>35 \mathrm{~kg} / \mathrm{m}^{2}$ (au lieu de $40 \mathrm{~kg} / \mathrm{m}^{2}$ en présence d'une obésité sans complications) $[11,12]$. Elles ne recommandent cependant pas cette approche chirurgicale lorsque l'IMC est inférieur à $35 \mathrm{~kg} / \mathrm{m}^{2}$. Pourtant, de nombreux progrès ont été réalisés au cours des dernières années quant à la compréhension des mécanismes hormono-métaboliques, expliquant les effets positifs de la chirurgie en ce qui concerne le contrôle du poids et l'homéostasie glycémique, indépendamment des effets mécaniques de restriction gastrique et de malabsorption intestinale $[5,6]$. Cette nouvelle dynamique ouvre des perspectives pour le traitement du DT2 [13].

Nous avons analysé, dans un précédent article, les effets hormono-métaboliques induits par les différents types de chirurgie bariatrique et discuté brièvement leurs impacts respectifs sur la régulation glycémique [6]. Les buts principaux de ce second article sont de rapporter et discuter les résultats des études les plus récentes ayant testé une solution chirurgicale chez le patient DT2 avec un $\mathrm{IMC}<35 \mathrm{~kg} / \mathrm{m}^{2}$. Nous conclurons en présentant un nouveau paradigme avec l'évolution d'une chirurgie bariatrique vers une chirurgie métabolique et spéculerons sur l'avenir de ce type de nouvelle approche dans le traitement du DT2.

\section{Études cliniques chez les patients DT2 avec IMC $<35 \mathrm{~kg} / \mathrm{m}^{2}$}

Au cours des dernières années, plusieurs équipes ont proposé des opérations de divers types à des patients DT2 présentant un IMC $<35 \mathrm{~kg} / \mathrm{m}^{2}[13,14]$. Cette évolution a été la résultante de deux constatations :

- d'une part, la chirurgie bariatrique a fourni des résultats spectaculaires chez le patient DT2 obèse, avec une résolution du DT2 dans 50 à $80 \%$ des cas. Cette amélioration s'opère très rapidement, avant même l'obtention d'une perte de poids importante, et était présente quel que soit le degré d'obésité de départ ;

- d'autre part, les progrès dans les connaissances physiopathologiques, évoquées ci-dessus et rapportées en détail par ailleurs [6], ont fait clairement apparaître que l'amélioration métabolique observée pouvait s'expliquer davantage par les modifications entérohormonales induites par la chirurgie plutôt que par la simple restriction ou la malabsorption alimentaire, laissant suspecter également un effet positif chez des patients DT2, indépendamment de la présence d'une obésité $[5,6]$.

Une revue systématique récente des études publiées entre 1979 et 2009 a identifié 16 études, rétrospectives ou prospectives, comportant un total de 343 patients DT2 avec un IMC $<35 \mathrm{~kg} / \mathrm{m}^{2}$ et ayant bénéficié de pas moins de huit procédures chirurgicales différentes [14]. II faut noter qu'en très grande majorité (en fait 11 sur 16), les études répertoriées proviennent d'Italie ou du Brésil. Après un suivi très variable selon les études (de 2 à 216 mois), I'IMC a diminué en moyenne de 29,4 à $24,2 \mathrm{~kg} / \mathrm{m}^{2}$. Au total, $85 \%$ des patients DT2 ont pu être sevrés des médicaments hypoglycémiants, atteignant une glycémie à jeun de 105 mg/dl (soit une diminution moyenne de $93 \mathrm{mg} / \mathrm{dl}$ par rapport à la valeur précédant l'opération) et un taux d'hémoglobine glyquée $\left(\mathrm{HbA}_{1 \mathrm{c}}\right)$ de $6 \%$ (soit une baisse moyenne de $2,7 \%)$. Ces résultats remarquables ont été obtenus avec un très faible de taux de complications (avec cependant une 
mortalité opératoire de $0,29 \%)$. Les auteurs concluent que ces observations ouvrent la porte à la chirurgie métabolique pour des patients DT2 avec un IMC $<30 \mathrm{~kg} / \mathrm{m}^{2}$.

Les techniques le plus anciennement testées chez les patients DT2 avec un IMC $<35 \mathrm{~kg} / \mathrm{m}^{2}$ ont fait appel à une méthode restrictive pure comme le cerclage gastrique ajustable ou, inversement, à une approche malabsorptive pure comme la dérivation bilio-pancréatique développée par Scopinaro. Nous rappellerons d'abord brièvement les résultats obtenus avec ces deux techniques. Ensuite, nous décrirons les résultats obtenus avec la dérivation gastrique avec anse de Roux en $\mathrm{Y}$, la méthode la plus utilisée en chirurgie bariatrique, notamment aux Etats-Unis (figure 1) ; elle représente, en fait, une combinaison de ces deux approches, avec une modification composite de la réponse endocrine intestinale [6]. Enfin, nous focaliserons notre attention sur les travaux faisant appel à des techniques chirurgicales tentant d'exploiter les trois grands mécanismes hormonométaboliques précédemment décrits, ciblant spécifiquement l'estomac (sleeve, ou gastrectomie en manchon), l'intestin proximal (exclusion duodénojéjunale) et l'intestin distal (interposition iléale) [6].

\section{Cerclage ajustable}

Le cerclage gastrique ajustable est une technique purement restrictive, apparemment simple, peu invasive et plus facilement réversible, et se distingue en cela de la dérivation gastrique avec anse de Roux en Y. Cet avantage a popularisé cette technique, plus particulièrement en Europe occidentale, dont la France et la Belgique. Réservée officiellement à des patients avec un IMC $>35 \mathrm{~kg} / \mathrm{m}^{2}$, cette approche a néanmoins été réalisée de façon plus exceptionnelle chez des patients avec un IMC $<35 \mathrm{~kg} / \mathrm{m}^{2}$. Ainsi, dans notre série de 658 patients publiée en 1998, 8,5\% des patients avaient bénéficié de cette intervention alors qu'ils n'atteignaient pas ce seuil critique d'IMC, avec néanmoins une

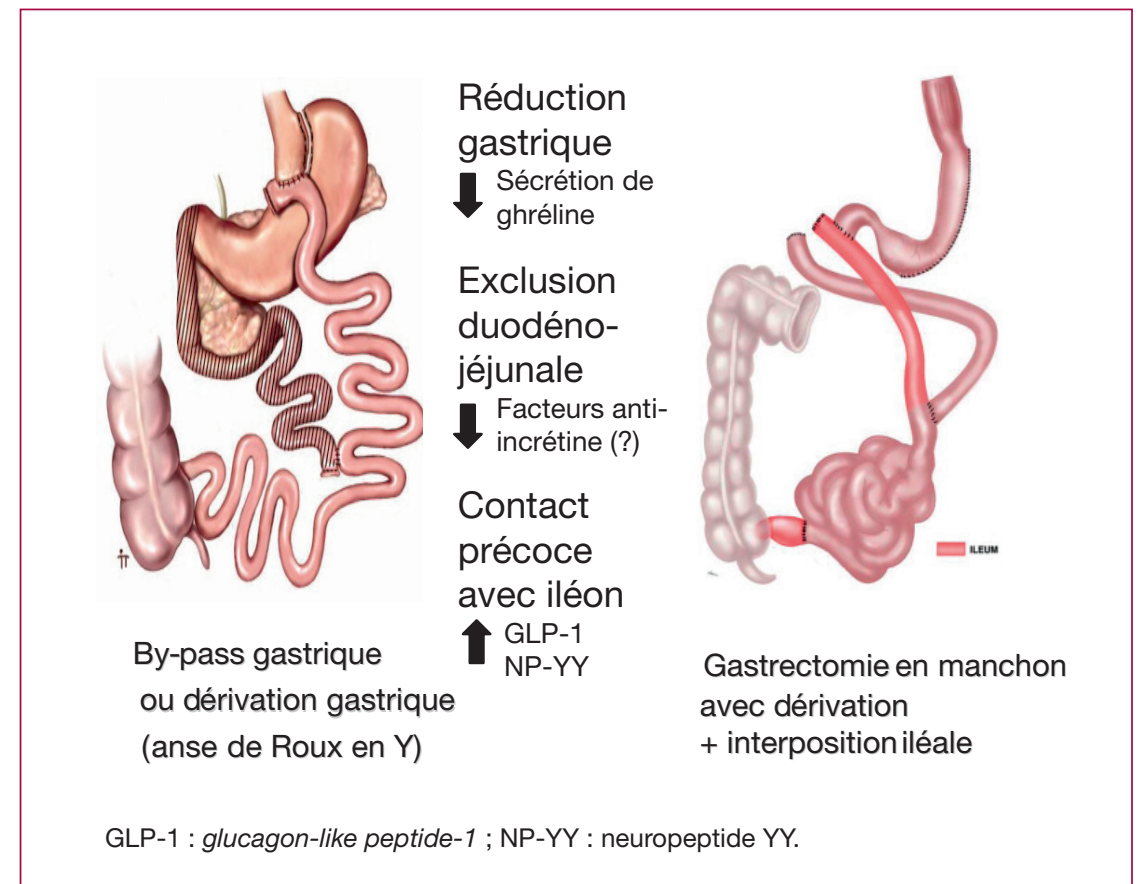

Figure 1 : Illustration de la dérivation gastrique avec anse de Roux en $Y$ (à gauche) et de la gastrectomie en manchon avec dérivation et interposition iléale (à droite), deux des principales techniques actuellement proposées par certaines équipes pour le traitement du diabète de type 2 chez des patients avec un IMC $<35 \mathrm{~kg} / \mathrm{m}^{2}$. Au milieu, description des principaux mécanismes impliqués dans l'amélioration métabolique. amélioration des divers marqueurs du syndrome métabolique, y compris la glycémie à jeun [15]. Depuis lors, au moins trois publications ont fait état de résultats positifs obtenus avec le cerclage gastrique ajustable chez des patients DT2 avec un IMC $<35 \mathrm{~kg} / \mathrm{m}^{2}$ [16-18] (tableau I). Dans un travail comparant le cerclage ajustable à un traitement médical conventionnel chez des patients DT2 avec un IMC entre 30 et $40 \mathrm{~kg} / \mathrm{m}^{2}$ (moyenne : $37,1 \mathrm{~kg} / \mathrm{m}^{2}$ ), seulement 13 des 60 patients inclus avaient un IMC $<35 \mathrm{~kg} / \mathrm{m}^{2}$, ce qui n'est guère représentatif [19]. Dans cette étude, I'IMC avant l'intervention n'était pas prédictif de la rémission du DT2, après ajustement pour la perte pondérale.

Au vu de l'importance des modifications hormono-métaboliques induites par les techniques de dérivation intestinale, susceptibles d'exercer des effets positifs sur l'homéostasie glycémique indépendamment de la perte de poids [6], il apparaît que le cerclage gastrique ajustable n'est pas la technique qui offre le plus d'opportunités chez le patient DT2 non obèse. Une étude comparative a d'ailleurs montré des résultats moins favorables avec le cerclage ajustable qu'avec le by-pass gastrique chez des patients DT2 avec un IMC compris entre 30 et $35 \mathrm{~kg} / \mathrm{m}^{2}$ [20] (tableau I).

\section{Dérivation bilio-pancréatique}

La technique de dérivation bilio-pancréatique a été popularisée par Scopinaro et al. [21]. II s'agit de la procédure chirurgicale qui entraîne la perte de poids la plus importante et le plus grand pourcentage de rémission du DT2 chez les patients avec obésité sévère. Cet effet s'explique non seulement par la malabsorption des aliments ingérés, mais également par les modifications hormonales induites par le court-circuit digestif [21]. Quelques publications ont rapporté des résultats positifs également chez des patients DT2 avec un IMC $<35 \mathrm{~kg} / \mathrm{m}^{2}$ [22-24] (tableau I).

Néanmoins, compte tenu des carences nutritionnelles que cette technique est susceptible de provoquer, la dérivation bilio-pancréatique ne paraît pas représenter la technique chirurgicale de premier choix chez le patient DT2 avec un $\mathrm{IMC}<35 \mathrm{~kg} / \mathrm{m}^{2}$. 
Tableau I : Résultats obtenus dans la chirurgie métabolique dans quelques études récentes réalisées chez des patients diabétiques de type 2 (DT2), avec un indice de masse corporelle $(\mathrm{IMC})<35 \mathrm{~kg} / \mathrm{m}^{2}$. La rémission est définie ici par l'arrêt complet de tout traitement hypoglycémiant.

\begin{tabular}{|c|c|c|c|c|c|c|c|c|c|}
\hline Référence & Technique & $\begin{array}{c}n \text { DT2 } \\
\text { avec suivi } \\
>6 \text { mois }\end{array}$ & $\begin{array}{c}\text { IMC } \\
\text { initial } \\
\left(\mathbf{k g} / \mathbf{m}^{2}\right)\end{array}$ & $\begin{array}{l}\text { IMC final } \\
\left(\mathbf{k g} / \mathbf{m}^{2}\right)\end{array}$ & $\begin{array}{c}\text { Glucose } \\
\text { initial } \\
\text { (mg/dl) }\end{array}$ & $\begin{array}{c}\text { Glucose } \\
\text { final } \\
\text { (mg/dl) }\end{array}$ & $\begin{array}{c}\mathrm{HbA}_{1 \mathrm{c}} \\
\text { initiale } \\
(\%)\end{array}$ & $\begin{array}{c}\mathrm{HbA}_{1 \mathrm{c}} \\
\text { finale } \\
(\%)\end{array}$ & $\begin{array}{c}\text { Taux de } \\
\text { rémission } \\
\text { (\%) }\end{array}$ \\
\hline 16 & LAGB & 4 & 33,9 & $29,7^{*}$ & ND & $\mathrm{ND}$ & ND & $\mathrm{ND}$ & 100 \\
\hline 17 & LAGB & 8 & 32,7 & $27,6^{*}$ & ND & ND & ND & ND & 100 \\
\hline 18 & LAGB & 15 & 33,1 & $25,8^{*}$ & ND & ND & ND & ND & 50 \\
\hline 20 & LAGB & 40 & 33,9 & 30,9 & ND & ND & ND & ND & 27,5 \\
\hline 22 & DBP & 10 & 33,3 & 27,5 & ND & ND & ND & ND & 100 \\
\hline 23 & DBP & 5 & 30,9 & 25,1 & ND & ND & 8,5 & 5,7 & 100 \\
\hline 24 & DBP & 7 & 30,6 & 25,3 & 220 & 136 & 9,3 & 6,3 & 100 \\
\hline 26 & GB & 37 & 32,5 & $N D^{* *}$ & 146 & 88 & ND & $\leq 6$ & 100 \\
\hline 27 & GB & 44 & 31,7 & 23,2 & 169 & 89 & 7,3 & 5,6 & ND \\
\hline 28 & GB & 15 & 28,9 & 23,0 & 233 & 89 & 10,1 & 6,1 & 100 \\
\hline 20 & GB & 29 & 33,7 & 27,2 & ND & ND & ND & ND & 55 \\
\hline 31 & DDJ & 2 & 29,6 & 28,3 & ND & 92 & ND & 5,35 & 100 \\
\hline 32 & DDJ & 12 & 26,1 & 25,6 & 185 & 158 & 8,9 & 7,8 & 0 \\
\hline 33 & DDJ & 20 & 27,1 & 24,4 & 171 & 96 & 8,8 & 6,8 & 90 \\
\hline 34 & DDJ & 7 & 27,5 & 27,3 & 209 & 155 & 9,4 & 8,5 & 14 \\
\hline 46 & $D S G+\|$ & 69 & 25,7 & 21,8 & 218 & 102 & 8,7 & 5,9 & 96 \\
\hline 47 & (D)SG + II & 58 & 28,2 & ND & 215 & 105 & 8,9 & 5,4 & $N D^{\star \star \star}$ \\
\hline 48 & $D S G+\|$ & 20 & 29 & 22,7 & ND & ND & 8,4 & 5,4 & ND \\
\hline 48 & $S G+\|$ & 18 & 27 & 22,2 & ND & ND & 8,6 & 6,35 & ND \\
\hline 51 & $S G+I I$ & 5 & 33,1 & 24,6 & 208 & 98 & 10,9 & 6,1 & 100 \\
\hline
\end{tabular}

$N D$ : non disponible; $L A G B$ : cerclage gastrique ajustable ; DBP : dérivation bilio-pancréatique ; GB : By-pass gastrique (avec anse de Roux en Y) ; $D D J$ : dérivation duodéno-jéjunale; DSG + II: Sleeve gastrectomie avec dérivation + interposition iléale; SG + II: Sleeve gastrectomie sans dérivation + interposition iléale ; * Les IMC initiaux et finaux de ces trois études correspondent à la population globale (diabétique et non diabétique) ; ** Perte de $78 \%$ de l'excès de poids initial ; ** Proportion de 91,2\% avec taux d'HbA 1 c $7 \%$ (avec ou sans traitement).

Dérivation gastrique avec anse de Roux en $Y$

La technique chirurgicale qui a été la plus utilisée (notamment aux États-Unis) et la mieux évaluée est, de loin, le by-pass gastrique, à savoir la dérivation gastrique avec anse de Roux en Y [25]. Cette technique combine des effets composites, sur l'estomac, sur l'intestin proximal et sur l'intestin distal, qui, tous trois, peuvent contribuer à améliorer le contrôle glycémique [6], comme cela a été amplement démontré chez le patient obèse DT2 [25]. II n'est donc pas étonnant que la dérivation gastrique avec anse de Roux en $\mathrm{Y}$ ait été testée chez des patients DT2 avec un IMC < 35 kg/m² [26-28] (tableau I).

- Dans une série brésilienne de 37 patients DT2 avec un IMC moyen de $32,5 \mathrm{~kg} / \mathrm{m}^{2}$, la perte moyenne de l'excès de poids initial a été de $81 \%, 6$ à 48 mois après le by-pass gastrique, avec une disparition des co-morbidités, dont le DT2, dans 36 cas [26].

- Une étude chinoise a comparé les effets de la dérivation gastrique réali- sée sous laparoscopie chez des patients DT2 avec un IMC < ou $>35 \mathrm{~kg} / \mathrm{m}^{2}$ [27]. Parmi 201 patients hyperglycémiques, 44 (22\%) avaient un IMC $<35 \mathrm{~kg} / \mathrm{m}^{2}$. Un an après la chirurgie, la glycémie à jeun est normalisée chez $89,5 \%$ des patients avec un IMC $<35 \mathrm{~kg} / \mathrm{m}^{2}$, taux un peu moins élevé que chez les patients avec un IMC > $35 \mathrm{~kg} / \mathrm{m}^{2}$ (98,5\%). Une différence comparable est observée entre les deux sous-groupes si l'on prend en compte les sujets récupérant un taux d'HbA $\mathrm{H}_{1 \mathrm{c}}<7 \%$ avec un bilan lipidique normal (76,5 versus 92,4\%). Ainsi, même si les résultats sont un peu moins spectaculaires chez les patients avec un IMC $<35 \mathrm{~kg} / \mathrm{m}^{2}$, ils n'en demeurent pas moins remarquables dans cette population asiatique.

- Enfin, dans une série limitée de 15 patients DT2 indiens asiatiques avec un IMC initial de $28,9 \mathrm{~kg} / \mathrm{m}^{2}$ et une durée connue moyenne de DT2 de 8,7 années ( $80 \%$ traités par insuline et $20 \%$ par antidiabétiques oraux), le by-pass gastrique a fait diminuer la glycémie à jeun de 233 à $89 \mathrm{mg} / \mathrm{l}$ et le taux d'HbA $\mathrm{A}_{1 \mathrm{c}}$ de
10,1 à $6,1 \%, 9$ mois après l'intervention, alors que tous les médicaments hypoglycémiants avaient été arrêtés dès le $3^{\mathrm{e}}$ mois [28].

- Une étude comparative d'envergure, comprenant 109 patients DT2 avec un IMC entre 30 et $35 \mathrm{~kg} / \mathrm{m}^{2}$ dans chaque groupe, a montré que la dérivation gastrique avec anse de Roux en Y, a entraîné une perte de poids supérieure et un taux de rémission du diabète plus élevé 6 à 12 mois après l'intervention, mais avec une incidence de complications plus importante durant les 3 premiers mois. Le suivi à long terme (> $1 \mathrm{an}$ ) reste cependant confiné à un nombre limité de patients dans ce travail [20] (tableau I).

Tous ces résultats sont encourageants et méritent certainement d'être confirmés dans de plus grandes séries suivies pendant une période plus prolongée.

\section{Exclusion duodéno-jéjunale}

II y a une dizaine d'années déjà, Pories et Albrecht avaient attiré l'attention sur le rôle de l'intestin proximal dans la 
physiopathologie du DT2 [29]. Depuis lors, diverses expériences animales et observations humaines ont confirmé que l'exclusion de la partie proximale de l'intestin, évitant le contact précoce avec les aliments, empêchait la sécrétion de substances, non encore identifiées, capables d'entraver la sécrétion et/ou l'action de l'insuline [6, 30]. Plusieurs équipes ont démontré que l'exclusion duodéno-jéjunale permettait d'améliorer le contrôle glycémique du patient DT2 avec un IMC $<35 \mathrm{~kg} / \mathrm{m}^{2}$ [31-34] (tableau I).

Plutôt que de recourir à une intervention chirurgicale relativement complexe, une alternative récemment testée consiste à placer une prothèse endoluminale dans le duodénum et la partie proximale du jéjunum, permettant ainsi d'exclure cette partie proximale de l'intestin et donc d'éviter tout contact avec les aliments. Cette technique a été ponctuellement testée chez les patients DT2, mais le recul reste encore insuffisant pour pouvoir conclure [35].

Comme pour les autres techniques, elle devra être évaluée en termes d'efficacité, de sécurité, de durabilité et de coût.

Interposition ou transposition iléale

Le rôle croissant attribué aux hormones dites incrétines (GLP-1 [glucagon-like peptide-1] et GIP [glucose-dependent insulinotropic polypeptide], respectivement secrétées par les cellules entéroendocrines $L$ et $K$ de l'iléon) dans la physiopathologie du DT2 a ouvert la voie à des approches chirurgicales innovantes [6, 36].

II y a plus de 10 ans déjà, Mason avait montré que la transposition iléale permettait d'augmenter la sécrétion de GLP1 , ce qui pouvait avoir des effets positifs à la fois dans le traitement de l'obésité et du DT2 [37]. Depuis lors, cette hypothèse a été confirmée, amenant à proposer une technique d'interposition iléale pour le traitement du DT2, même en l'absence d'obésité importante [38, 39]. La transposition iléale, avec ou sans gastrectomie par laparoscopie, a été proposée comme la troisième génération de chirurgie bariatrique [40]. Cependant, la transposition iléale, visant à réaliser ce que certains appellent un « frein neuro- endocrine " (neuroendocrine brake), n'est pas actuellement utilisée de façon isolée pour le traitement du DT2. En revanche, elle a été exploitée en combinaison avec une gastrectomie en manchon, avec ou sans dérivation (voir ci-dessous).

\section{Gastrectomie en manchon} combinée à une interposition iléale La gastrectomie en manchon (sleeve gastrectomy) a été récemment utilisée dans le traitement du patient avec DT2 [4143]. Cependant, cette opération a surtout été réalisée chez des patients obèses. Elle n'a donc pas été évaluée de façon isolée chez le patient DT2 avec un IMC $<35 \mathrm{~kg} / \mathrm{m}^{2}$. Une étude est actuellement en cours comparant la gastrectomie en manchon avec le cerclage ajustable chez 150 patients DT2 avec un IMC compris entre 27 et $42 \mathrm{~kg} / \mathrm{m}^{2}$, suivis pendant une année, avec comme critère d'évaluation principal la récupération d'un taux d'HbA $_{1 \mathrm{c}}<6 \%$ [44].

La gastrectomie en manchon a été combinée avec la procédure d'interposition iléale par une équipe brésilienne qui a publié plusieurs articles rapportant les résultats d'une série de patients DT2, de plus en plus nombreux, de 2008 à 2010, et/ou avec une durée de suivi de

\section{Les points essentiels}

- Le diabète de type 2 (DT2) est une maladie plus complexe qu'escomptée initialement, qui, dans bien des cas, reste imparfaitement contrôlée par les traitements médicaux classiques.

- La meilleure compréhension des mécanismes impliqués dans l'amélioration métabolique accompagnant la chirurgie bariatrique a ouvert de nouvelles perspectives en faveur d'une solution chirurgicale pour le traitement du DT2, même en l'absence d'une obésité importante associée.

- Plusieurs techniques chirurgicales ont été évaluées chez des patients DT2 avec un $\mathrm{IMC}<35 \mathrm{~kg} / \mathrm{m}^{2}$, et même $<30 \mathrm{~kg} / \mathrm{m}^{2}$ : restrictives pures (cerclage gastrique ajustable), malabsorptives pures (dérivation bilio-pancréatique) ou mixtes (dérivation gastrique avec anse de Roux en Y).

- L'avenir réside peut-être en des techniques innovantes combinant une restriction gastrique (gastrectomie en manchon), une exclusion duodéno-jéjunale (éventuellement avec mise en place d'une endoprothèse) et/ou une amplification des effets intestinaux distaux (interposition iléale).

- Avant de progresser d'une chirurgie bariatrique vers une chirurgie métabolique et de considérer ce nouveau paradigme pour le traitement du DT2 sans obésité avérée, il faut poursuivre une évaluation soigneuse du rapport bénéfices/risques des différentes techniques proposées, non seulement à court terme, mais également à long terme, en ce incluse une analyse pharmaco- et socio-économique. 
L'opération décrite par DePaula et al. est, en fait, très proche d'une diversion biliopancréatique avec un duodenal switch, tout en étant plus complexe sur le plan chirurgical et donc à plus haut risque théorique de complications, comme souligné récemment par Gagner [52, 53]. Néanmoins, DePaula et al. ont publié récemment la morbi-mortalité à 30 jours d'une grande série de 454 patients DT2 avec un IMC $<35 \mathrm{~kg} / \mathrm{m}^{2}$ [54]. Les auteurs concluent que l'opération est sûre, même s'ils rapportent une mortalité de $0,4 \%$, un taux de complications majeures de $6,4 \%$ et un taux de complications mineures de $11,2 \%$.

Par comparaison à la classique dérivation gastrique avec anse de Roux en $Y$, cette procédure est sensée se montrer encore plus efficace sur le plan de l'homéostasie glycémique. En effet, ainsi que discuté dans un article précédent [6], la gastrectomie en manchon diminue davantage les taux de ghréline que le by-pass gastrique et l'interposition d'une anse iléale à un niveau plus proximal devrait encore potentialiser davantage la réponse incrétine en GLP-1 [6]. À notre connaissance, il n'existe cependant pas d'études publiées comparant directement l'efficacité et la sécurité de ces deux approches chirurgicales.

\section{De la chirurgie bariatrique à la chirurgie métabolique}

On est en train d'assister, manifestement, à une évolution de la chirurgie du patient avec DT2, passant de la chirurgie bariatrique classique, réservée aux sujets avec une obésité sévère (IMC $>35 \mathrm{~kg} / \mathrm{m}^{2}$, voire $\left.>40 \mathrm{~kg} / \mathrm{m}^{2}\right)[1-4,10]$ à une chirurgie métabolique, proposée à des personnes sans obésité marquée, avec un IMC $<35 \mathrm{~kg} / \mathrm{m}^{2}$, voire $<30 \mathrm{~kg} / \mathrm{m}^{2}$ [5, 13, 14, 55, 56].

Le DT2 est une maladie complexe, combinant déficits de l'insulinosécrétion et de l'insulinosensibilité. II est caractérisé par une dégradation progressive du contrôle glycémique au cours du temps, liée à une perte inéluctable (jusqu'à présent) de la capacité insulinosécrétoire de la cellule $\beta$. Par ailleurs, il est associé à de nombreux autres facteurs de risque cardiovasculaire, ce qui explique l'incidence élevée et la forte prévalence des accidents coronariens et cérébrovasculaires. L'escalade thérapeutique qui s'en suit pour contrôler au mieux la glycémie et la polymédication pour corriger les nombreux facteurs de risque n'empêche pas le fait que les patients DT2 restent exposés à un risque élevé de complications et de décès prématuré. Force est donc de reconnaître que les approches médicales sont grevées de lacunes non négligeables [7].

Dès 1992, Pories et al. mentionnaient, de façon provocante à l'époque, il est vrai, que le DT2 est une maladie « chirurgicale " [57] et, trois années plus tard, ils renchérissaient en proclamant « qui aurait

\section{Conclusion : vers un nouveau paradigme?}

La mutation de la chirurgie bariatrique en chirurgie métabolique $[66,67]$ ouvre la voie à un nouveau paradigme dans le traitement du DT2, l'option d'une solution chirurgicale en cas d'échec du traitement médical, même en l'absence d'obésité [13]. On se trouve sans doute actuellement à un croisement dans la prise en charge du DT2 :

- soit les nouveaux traitements pharmacologiques proposés permettent d'améliorer drastiquement la situation souvent largement imparfaite des patients DT2

- soit le traitement médical demeure un échec ouvrant alors la porte à la chirurgie métabolique comme alternative [64].

Par ailleurs, il ne fait aucun doute que l'adhésion à une meilleure hygiène de vie représente la base même du traitement du DT2, a fortiori s'il existe un certain excès de poids. Force est de constater cependant qu'adhérer au long cours aux conseils nutritionnels est une tâche quasi impossible pour ces patients, de telle sorte qu'une aide chirurgicale pourrait représenter une aide appréciable, voire, dans certains cas, la véritable solution au problème [68].

Quoi qu'il en soit, avant d'adhérer à ce nouveau paradigme, il convient de disposer d'études plus larges, non limitées à quelques équipes, bien contrôlées et couvrant une période suffisamment longue de façon à pouvoir disposer d'une évaluation objective quant au rapport bénéfices/risques de ce type d'approche. Une analyse pharmaco- et socio-économique est également indispensable avant que les compagnies d'assurance, publiques ou privées, acceptent le remboursement de ce type d'intervention chez des patients DT2 avec un IMC $<35 \mathrm{~kg} / \mathrm{m}^{2}[61,69]$. Une tentative d'analyse de ce type a été entreprise récemment, mais n'a pris en considération que la technique du cerclage ajustable qui, nous l'avons vu, ne paraît pas être la plus performante chez le patient DT2 [69]. On attend également une estimation tant de la qualité de vie que de l'espérance de vie (cette dernière requiert un recul suffisant) chez les patients DT2 traités par chirurgie métabolique plutôt que par traitement médical conventionnel.

Rejeter d'emblée la chirurgie métabolique ne paraît guère justifié au vu des progrès récents dans la compréhension des mécanismes hormono-métaboliques sous-jacents et dans les techniques chirurgicales proposées. À l'inverse, se précipiter vers cette solution chirurgicale semble à tout le moins prématuré et ne paraît pas raisonnable en l'état actuel [60]. La devise "Wait and see " s'impose donc, mais en gardant une ouverture d'esprit excluant a priori tout ostracisme. On est, en effet, peut-être à l'aube d'une véritable révolution dans la prise en charge du DT2 ! 
L'une des questions fondamentales, qui se pose déjà actuellement et qui se posera de plus en plus si la chirurgie métabolique prend son essor, est de savoir à quel patient DT2 proposer une solution chirurgicale [61]. Actuellement, il paraît raisonnable de réserver cette chirurgie en priorité aux patients DT2 les plus obèses, car ce sont sans doute eux qui tireront globalement le plus de bénéfices de l'intervention [61]. II ne fait cependant pas de doute que l'on assiste à un changement de cap, ouvrant la porte à des patients DT2 sans obésité sévère, voire même avec un simple excès de poids [13]. Cette approche chirurgicale pourrait avoir une place particulière dans la population asiatique qui, on le sait, est confrontée à un risque élevé de DT2 dès que l'IMC est modérément élevé (par exemple $>25 \mathrm{~kg} / \mathrm{m}^{2}$ ) [62]. Certains ont cependant mis en garde contre un engouement excessif pour la chirurgie métabolique chez les patients DT2 non obèses [60,63]. Le risque hypoglycémique, dû à une sécrétion excessive d'insuline, a été mis en avant par certains, même s'il parait assez exceptionnel [64]. Par ailleurs, plus on s'intéresse à des patients diabétiques avec un $\mathrm{IMC}<30 \mathrm{~kg} / \mathrm{m}^{2}$, plus il existe un risque de sélectionner des patients avec un diabète de type 1 lent plutôt qu'avec un DT2 vrai [65].

Enfin, il faut tenir compte des complications toujours possibles inhérentes à l'opération [54, 60]. Celles-ci doivent être mises en balance avec le risque naturel associé au DT2. À ce sujet, il ne faut sans doute pas négliger le fait que les progrès du traitement médical, grâce notamment à une approche globale intégrée, ont contribué à réduire substantiellement le risque cardiovasculaire des patients DT2 au cours des dernières années [9].

\section{Déclaration d'intérêt}

Les auteurs déclarent ne pas avoir de conflit d'intérêt en relation avec cet article.

\section{Références}

[1] Dixon JB. Obesity and diabetes: the impact of bariatric surgery on type-2 diabetes. World J Surg 2009;33:2014-21.

[2] Vetter ML, Cardillo S, Rickels MR, Iqbal N. Narrative review: effect of bariatric surgery on type 2 diabetes mellitus. Ann Intern Med 2009;150:94-103.

[3] Scheen AJ, De Flines J, De Roover A, Paquot $N$. Bariatric surgery in patients with type 2 diabetes: benefits, risks, indications and perspectives. Diabetes Metab 2009;35:537-43.

[4] Buchwald H, Estok R, Fahrbach H, et al. Weight and type 2 diabetes after bariatric surgery: systematic review and meta-analysis. Am J Med 2009;122:248-56.

[5] Rubino F, Schauer PR, Kaplan LM, Cummings DE. Metabolic surgery to treat type 2 diabetes: clinical outcomes and mechanisms of action. Annu Rev Med 2010;61:393-411.

[6] Scheen AJ, De Flines J, De Roover A, Paquot N. Le tractus digestif comme organe endocrine: une nouvelle vision de la chirurgie bariatrique. Med Mal Metab 2011;5: in press.

[7] Scheen AJ. Current management strategies for coexisting diabetes mellitus and obesity. Drugs 2003;63:1165-84.

[8] Esser N, Paquot N, Scheen AJ. Aptitude physique versus adiposité : impacts métaboliques respectifs chez le sujet avec une diminution de la tolérance au glucose ou un diabète de type 2 . Med Mal Metab 2010;4:673-80.

[9] Scheen AJ, Paquot N. Quelle est la nouvelle donne pour soigner les patients diabétiques de type 2 ? Med Mal Metab 2009;3:141-6.

[10] Scheen AJ. Aggressive weight reduction treatment in the management of type 2 diabetes. Diabetes Metab 1998;24:116-23.

[11] National Institutes of Health Consensus Development Panel. Gastrointestinal surgery for severe obesity. Consensus Development Conference Statement. Ann Intern Med 1991;115:956-61.

[12] Fried M, Hainer V, Basdevant A, et al.; Bariatric Scientific Collaborative Group Expert Panel. Interdisciplinary European guidelines for surgery for severe (morbid) obesity. Obes Surg 2007;17:260-70.

[13] Rubino F, Kaplan LM, Schauer PR, Cummings DE; Bariatric Scientific Collaborative Group Expert Panel. The Diabetes Surgery Summit consensus conference: recommendations for the evaluation and use of gastrointestinal surgery to treat type 2 diabetes mellitus. Ann Surg 2010;251:399-405.

[14] Fried M, Ribaric G, Buchwald JN, et al. Metabolic surgery for the treatment of type 2 diabetes in patients with $\mathrm{BMI}<35 \mathrm{~kg} / \mathrm{m}^{2}$ : an integrative review of early studies. Obes Surg 2010;20:776-90.

[15] Luyckx FH, Scheen AJ, Desaive C, et al. Effects of gastroplasty on body weight and related biological abnormalities in morbid obesity. Diabetes Metab 1998;24:355-61.

[16] Angrisani L, Favretti F, Furbetta F, et al. Italian Group for Lap-Band System ${ }^{\circledR}$ : results of multicenter study on patients with $\mathrm{BMI} \leq 35 \mathrm{~kg} / \mathrm{m}^{2}$. Obes Surg 2004;14:415-8.

[17] Parikh M, Duncombe J, Fielding GA. Laparoscopic adjustable gastric banding for patients with body mass index of $\leq 35 \mathrm{~kg} / \mathrm{m}^{2}$. Surg Obes Rel Dis 2006;2:518-22.

[18] Sultan S, Parikh M, Youn H, et al. Early U.S. outcomes after laparoscopic adjustable gastric banding in patients with a body mass index less than 35 kg/m². Surg Endosc 2009;23:1569-73.

[19] Dixon JB, O'Brien PE, Playfair J, et al. Adjustable gastric banding and conventional therapy for type 2 diabetes: a randomized controlled trial. JAMA 2008;299:316-23.

[20] Demaria EJ, Winegar DA, Pate VW, et al. Early postoperative outcomes of metabolic surgery to treat diabetes from sites participating in the ASMBS bariatric surgery center of excellence program as reported in the Bariatric Outcomes Longitudinal Database. Ann Surg 2010;252:55966; discussion 566-7.

[21] Scopinaro N, Papadia F, Camerini G, et al. A comparison of a personal series of biliopancreatic diversion and literature data on gastric bypass help to explain the mechanisms of resolution of type 2 diabetes by the two operations. Obes Surg 2008;18:1035-8.

[22] Noya G, Cossu ML, Coppola M, et al Biliopancreatic diversion preserving the stomach and pylorus in the treatment of hypercholesterolemia and diabetes type II: results in the first 10 cases. Obes Surg 1998;8:67-72.

[23] Chiellini C, Rubino F, Castagneto M, et al. The effect of bilio-pancreatic diversion on type 2 diabetes in patients with BMl $<35 \mathrm{~kg} / \mathrm{m}^{2}$. Diabetologia 2000;52:1027-30.

[24] Scopinaro N, Papadia F, Marinari G, et al. Long-term control of type 2 diabetes mellitus and the other major components of the metabolic syndrome after biliopancreatic diversion in patients with BMI $<35 \mathrm{~kg} / \mathrm{m}^{2}$. Obes Surg 2007;17:185-92.

[25] Cummings DE. Endocrine mechanisms mediating remission of diabetes after gastric bypass surgery. Int J Obes (Lond) 2009;33(Suppl.1):S33-40. [26] Cohen R, Pinheiro JS, Correa JL, Schiavon CA. Laparoscopic Roux-en-Y gastric bypass for BMI $<35 \mathrm{~kg} / \mathrm{m}^{2}$ : a tailored approach. Surg Obesity Relat Dis 2006;2:401-4; discussion 404.

[27] Lee WJ, Wang W, Lee YC, et al. Effect of laparoscopic mini-gastric bypass for type 2 diabetes mellitus: comparison of $\mathrm{BMl}>35$ and $<35 \mathrm{~kg} / \mathrm{m}^{2}$. J Gastrointest Surg 2008;12:945-52.

[28] Shah SS, Todkar JS, Shah PS, Cummings DE. Diabetes remission and reduced cardiovascular risk after gastric bypass in Asian Indians with body mass index $<35 \mathrm{~kg} / \mathrm{m}^{2}$. Surg Obes Relat Dis 2010;6:332-8.

[29] Pories WJ, Albrecht RJ. Etiology of type II diabetes mellitus: role of the foregut. World $\mathrm{J}$ Surg 2001;25:527-31.

[30] Knop FK. Resolution of type 2 diabetes following gastric bypass surgery: involvement of gut-derived glucagon and glucagonotropic signalling? Diabetologia 2009;52:2270-6.

[31] Cohen RV, Schiavon CA, Pinheiro JS, et al. Duodenal-jejunal bypass for the treatment of type 2 diabetes in patients with body mass index of $22-34 \mathrm{~kg} / \mathrm{m}^{2}$ : a report of 2 cases. Surg Obes Relat Dis 2007;3:195-7.

[32] Geloneze B, Geloneze SR, Fiori C, et al. Surgery for nonobese type 2 diabetic patients: an interventional study with duodenal-jejunal exclusion. Obes Surg 2009;19:1077-83.

[33] Ramo AC, Galvão Neto MP, de Souza YM, et al. Laparoscopic duodenal-jejunal exclusion 
in the treatment of type 2 diabetes mellitus in patients with $\mathrm{BMl}<30 \mathrm{~kg} / \mathrm{m}^{2}$ (LBMI). Obes Surg 2009;19:307-12.

[34] Ferzli GS, Dominique E, Ciaglia M, et al. Clinical improvement after duodenojejunal bypass for nonobese type 2 diabetes despite minimal improvement in glycemic homeostasis. World $\mathrm{J}$ Surg 2009;33:972-9.

[35] Rodriguez L, Reyes E, Fagalde P, et al. Pilot clinical study of an endoscopic, removable duodenal-jejunal bypass liner for the treatment of type 2 diabetes. Diabetes Technol Ther 2009;11:725-32.

[36] Gutierrez-Aguilar R, Woods SC. Nutrition and $L$ and K-enteroendocrine cells. Curr Opin Endocrinol Diabetes Obes 2011;18:35-41.

[37] Mason EE. lleal transposition and enteroglucagon/GLP-1 in obesity (and diabetic?) surgery. Obes Surg 1999;9:223-8.

[38] Strader AD. Ileal transposition provides insight into the effectiveness of gastric bypass surgery. Physiol Behav 2006;88:277-82.

[39] Ahima RS, Carr R. Alas! lleal interposition surgery for diabetes prevention? Gastroenterology 2010;138:2224-6.

[40] Gagner M. La transposition iléale avec ou sans gastrectomie par laparoscopie chez l'homme (TIG) : la troisième génération de chirurgie bariatrique. J Cœliochirurgie 2005;54:4-10.

[41] Rosenthal R, Li X, Samuel S, et al. Effect of sleeve gastrectomy on patients with diabetes mellitus. J Surg Obes Relat Dis 2009;5:429-34.

[42] Brethauer SA, Hammel JP, Schauer PR. Systematic review of sleeve gastrectomy as staging and primary bariatric procedure. Surg Obesity Relat Dis 2009;5:469-75.

[43] Gill RS, Birch DW, Shi X, et al. Sleeve gastrectomy and type 2 diabetes mellitus: a systematic review. Surg Obes Relat Dis 2010;6:707-13.

[44] Kashyap SR, Bhatt DL, Schauer PR; STAMPEDE Investigators. Bariatric surgery vs. advanced practice medical management in the treatment of type 2 diabetes mellitus: rationale and design of the Surgical Therapy And Medications Potentially Eradicate Diabetes Efficiently trial (STAMPEDE). Diabetes Obes Metab 2010;12:452-4.

[45] DePaula AL, Macedo AL, Rassi N, et al. Laparoscopic treatment of type 2 diabetes mellitus for patients with a body mass index less than 35. Surg Endosc 2008;22:706-16.
[46] DePaula AL, Macedo AL, Mota BR, Schraibman V. Laparoscopic ileal interposition associated to a diverted sleeve gastrectomy is an effective operation for the treatment of type 2 diabetes mellitus patients with BMI 21-29. Surg Endosc 2009;23:1313-20.

[47] DePaula AL, Macedo AL, Schraibman V, et al. Hormonal evaluation following laparoscopic treatment of type 2 diabetes mellitus patients with BMI 20-34. Surg Endosc 2009;23:1724-32.

[48] DePaula AL, Stival AR, Macedo A, et al. Prospective randomized controlled trial comparing 2 versions of laparoscopic ileal interposition associated with sleeve gastrectomy for patients with type 2 diabetes with BMI $21-34 \mathrm{~kg} / \mathrm{m}^{2}$. Surg Obes Relat Dis 2010;6:296-304.

[49] DePaula AL, Macedo AL, Rassi N, et al. Laparoscopic treatment of metabolic syndrome in patients with type 2 diabetes mellitus. Surg Endosc 2008;22:2670-8.

[50] DePaula AL, Stival AR, DePaula CC, et al. Impact on dyslipidemia of the laparoscopic ileal interposition associated to sleeve gastrectomy in type 2 diabetic patients. J Gastrointest Surg 2010;14:1319-25.

[51] Kumar KV, Ugale S, Gupta N, et al. Ileal interposition with sleeve gastrectomy for control of type 2 diabetes. Diabetes Technol Ther 2009;11:785-9.

[52] Gagner M. Surgical treatment of nonseverely obese patients with type 2 diabetes mellitus: sleeve gastrectomy with ileal transposition (SGIT) is the same as the neuroendocrine brake (NEB) procedure or ileal interposition associated with sleeve gastrectomy (II-SG), but ileal interposition with diverted sleeve gastrectomy (II-DSG) is the same as duodenal switch. Surg Endosc $2010 \mathrm{Jul}$ 8 [Epub ahead of print].

[53] Gagner M. Laparoscopic Sleeve Gastrectomy with lleal Interposition (SGIT): a modified duodenal switch for resolution of type 2 diabetes mellitus in lesser obese patients (BMI <35). World $\mathrm{J}$ Surg 2011;35:109-10.

[54] DePaula AL, Stival A, Halpern A, Vencio $\mathrm{S}$. Thirty-day morbidity and mortality of the laparoscopic ileal interposition associated with sleeve gastrectomy for the treatment of type 2 diabetic patients with $\mathrm{BMI}<35$ : an analysis of 454 consecutive patients. World J Surg 2011;35:102-8.

[55] Schulman AP, del Genio F, Sinha N, Rubino F. "Metabolic" surgery for treatment of type 2 diabetes mellitus. Endocr Pract 2009;15:624-31.
[56] Rubino F, R'bibo SL, del Genio F, et al. Metabolic surgery: the role of the gastrointestinal tract in diabetes mellitus.

[57] Pories WJ, MacDonald KG, Flickinger EG, et al. Is type II diabetes mellitus (NIDDM) a surgical disease? Ann Surg 1992;215:633-42; discussion 643.

[58] Pories WJ, Swanson MS, MacDonald KG, et al. Who would have thought it? An operation proves to be the most effective therapy for adultonset diabetes mellitus. Ann Surg 1995;222:33950; discussion 350-2.

59] Rubino F. Is type 2 diabetes an operable intestine disease? A provocative yet reasonable hypothesis. Diabetes Care 2008;31(suppl.2):S290-6.

[60] Giusti V. Chirurgie du diabète : provocation ou réalité ? Rev Med Suisse 2010;6:670-4; 676.

[61] Purnell JQ, Flum DR. Bariatric surgery and diabetes: who should be offered the option of remission? JAMA 2009;301:1593-5.

[62] Lakdawala M, Bhasker A; Asian Consensus Meeting on Metabolic Surgery (ACMOMS). Report: Asian Consensus Meeting on Metabolic Surgery. Recommendations for the use of Bariatric and Gastrointestinal Metabolic Surgery for Treatment of Obesity and Type II Diabetes Mellitus in the Asian Population: August $9^{\text {th }}$ and $10^{\text {th }}, 2008$, Trivandrum, India. Obes Surg 2010;20:929-36. [63] Deitel M. Surgery for diabetes at lower BMI: some caution. Obes Surg 2008;18:1211-4.

[64] Couzin J. Medicine. Bypassing medicine to treat diabetes. Science 2008;320:438-40.

[65] Deitel M. From bariatric to metabolic surgery in non-obese subjects: time for some caution. Arq Bras Endocrinol Metabol 2009;53:246-51.

[66] Chevallier JM. De la chirurgie de l'obésité à la chirurgie a visée métabolique. Expérience de quinze ans dans un service hospitalier universitaire. Bull Acad Natl Med 2010;194:25-36; discussion 36-8.

[67] DeMaria EJ. Announcing the arrival of metabolic surgery for diabetes. Ann Surg 2010;251:406-8.

[68] Magee C, Goenka N, Kerrigan D. Nutrition in type 2 diabetes. Metabolic surgery may be more effective. BMJ 2010 Aug 19 [Epub ahead of print].

[69] Villamizar N, Pryor AD. Safety, effectiveness, and cost effectiveness of metabolic surgery in the treatment of type 2 diabetes mellitus. J Obes 2011 [Epub 2010 Nov 14]. 